\title{
Supernovae data and perturbative deviation from homogeneity
}

\author{
Kari Enqvist* \\ Helsinki Institute of Physics, P.O.Box 64, FIN-00014 University of Helsinki, Finland; \\ Physics Department, P.O. Box 64, FIN-00014 University of Helsinki, Finland \\ Maria Mattsson ${ }^{\dagger}$ and Gerasimos Rigopoulos ${ }^{\ddagger}$ \\ Helsinki Institute of Physics, P.O.Box 64, FIN-00014 University of Helsinki, Finland
}

\begin{abstract}
We show that a spherically symmetric perturbation of a dust dominated $\Omega=1 \mathrm{FRW}$ universe in the Newtonian gauge can lead to an apparent acceleration of standard candles and provide a fit to the magnitude-redshift relation inferred from the supernovae data, while the perturbation in the gravitational potential remains small at all scales. We also demonstrate that the supernovae data does not necessarily imply the presence of some additional non-perturbative contribution by showing that any Lemaitre-Tolman-Bondi model fitting the supernovae data (with appropriate initial conditions) will be equivalent to a perturbed FRW spacetime along the past light cone.
\end{abstract}

\section{INTRODUCTION}

A basic principle which has enabled substantial progress in cosmology, referred to as the Copernican Principle, is the assumption that no observer has a privileged position in space. Coupled with the observed isotropy of the universe around us, this principle leads to the conclusion that the universe is homogeneous. Although the consistency of the current cosmological models strongly supports this idea, the Copernican Principle has never been explicitly tested; even the possibility of such testing has only been raised rather recently [1, 2, 3]. Even if the Copernican Principle is in general valid, there is still the possibility that it is mildly violated at some cosmologically significant level and that observers could occupy inequivalent spatial locations. In that case, their observations might mislead them into inferences about the universe as a whole and its global parameters which are incorrect.

Doubts about the validity of the Copernican Principle lie very much at the heart of some of the explanations put forward to account for the apparent acceleration implied by the Type I supernovae data [4]. As is well known, all cosmological data is consistent with a homogeneous $\Lambda \mathrm{CDM}$ concordance model, which assumes the existence of a cosmological constant $\Lambda$ that makes up about $70 \%$ of the current energy budget of the universe. Equally well acknowledged is the fact that, from a theoretical point of view, the incredibly small value of the required cosmological constant, about $10^{-120} M_{\mathrm{pl}}$, is a mystery. Moreover, there is also a peculiar coincidence that $\Lambda$ appears to have become important at the time when structures started to form. This may be taken as a hint towards the possibility that inhomogeneities could in some fashion be responsible for the apparent acceleration. One school of thought attempts to explain it as the influence, sometimes called backreaction, of averaging inhomogeneities on the average expansion of the universe [5, 6, 7, 8, 9, 10, 11, 12, 13]. Backreaction would be an inherently nonlinear effect due to the nonlinearity of general relativity. In this case the Copernican Principle could still hold in some statistical sense. Another possibility is to abandon the Copernican Principle altogether and assume that our location in the universe is exceptional, thereby fooling us into thinking that a cosmological constant exists, while in reality it is only a trick of the light. Such considerations have mostly been put forward within the context of spherically symmetric Lemaitre-Tolman-Bondi (LTB) models [14, 15, 16, 17]. Many authors have considered various possibilities offered by LTB models [18], which include inhomogeneities in the matter distribution as well as inhomogeneities in the expansion rate; an example is a dust universe with a local underdensity [19] that seems to be able to reproduce the SNIa observations.

There is an ongoing debate about the role of inhomogeneities, particularly whether the effects of inhomogeneities are too large to be describable within the standard perturbed FRW description. In contrast to various model calculations, in the present paper we do not make an attempt at model building and full-blown data fitting. Rather, we address the generic question of whether the SNIa data requires a deviation from the Copernican Principle that is nonperturbative. To explore this issue, we consider an FRW spacetime that has small perturbations in the gravitational potential $\psi$ (although the density perturbations could well be large). We assume an $\Omega=1$ dust universe and,

\footnotetext{
* E-mail: kari.enqvist@helsinki.fi

$\dagger$ E-mail: maria.ronkainen@helsinki.fi

¥ E-mail: gerasimos.rigopoulos@helsinki.fi
} 
for simplicity, spherically symmetric perturbations, and solve for $\psi$ and the velocity field $\mathbf{v}$ such that the distanceluminosity relation of the SNIa data is satisfied; this we do in Newtonian gauge. We find that the SNIa data can be fitted with $\psi$ and $\mathbf{v}$ remaining small during the whole evolution, meaning that the perturbed FRW framework works well and the Copernican Principle is thus only weakly violated in the sense that there are only small deviations from homogeneity. Furthermore, one might then argue that any effects of averaging over these small inhomogeneous perturbations, i.e. the backreaction, remain negligible [20]; due to the nonlinear nature of backreaction, the effects of averaging can only arise at second order in the perturbations and would hence be extremely small. Indeed, if the spacetime metric of the real universe were a perturbed FRW, it would imply that the backreaction is insignificant and thus could not solve the dark energy problem 9, 21]. However, we should note that the backreaction issue is somewhat more involved, and refer the reader to [9, 10, 21] and the references therein. Indeed, in a nonlinear theory there could arise additional non-perturbative contributions to metric perturbations which are not manifest at any order of perturbation theory performed about the FRW background but could be revealed by a careful comparison of data and results obtained from perturbation theory. As we will demonstrate, at least the supernovae data does not necessarily imply the presence of some additional non-perturbative contribution.

Finally, we contrast our result with LTB models, which under certain conditions are known to be physically equivalent to perturbed FRW models [9, 10, 22], although it has also been claimed that the LTB models capable of accounting for the SNIa data cannot be obtained from a perturbed FRW model [11]. Here we show explicitly that such conditions are fulfilled for realistic LTB models fitting the SNIa data and thus conclude that an explanation of the SNIa data in terms of (spherically symmetric) inhomogeneities does not require non-perturbative effects that would not be already present in a perturbed FRW description.

\section{A SPHERICAL PERTURBATION}

Let us assume that the spacetime can be described by a perturbed FRW model in the Newtonian gauge (vanishing anisotropic stress)

$$
d s^{2}=a^{2}\left[-(1+2 \psi) d \eta^{2}+(1-2 \psi) d \mathbf{x}^{2}\right]
$$

The effect of linear perturbations on the luminosity distance has been calculated in the past 23, 24, 25]. We follow here the notation of Bonvin, Durrer and Gasparini [25], who find the luminosity distance $d_{L}\left(\eta_{s}, \hat{\mathbf{n}}\right)$ of a source at conformal time $\eta_{s}$ in a universe described by the metric (11) as given by [25]

$$
\begin{aligned}
\frac{d_{L}\left(\eta_{s}, \hat{\mathbf{n}}\right)}{1+z}-\Delta \eta= & \left(\mathbf{v}_{o} \cdot \hat{\mathbf{n}}-\psi_{o}\right) \Delta \eta-2 \Delta \eta \mathbf{v} \cdot \hat{\mathbf{n}}+2 \int_{\eta_{s}}^{\eta_{o}} d \lambda \psi+2 \Delta \eta \int_{\eta_{s}}^{\eta_{o}} d \lambda \hat{\mathbf{n}} \cdot \nabla \psi \\
& +2 \int_{\eta_{s}}^{\eta_{o}} d \lambda \int_{\eta_{s}}^{\lambda} d \bar{\lambda} \hat{\mathbf{n}} \cdot \nabla \psi-\int_{\eta_{s}}^{\eta_{o}} d \lambda \int_{\eta_{s}}^{\lambda} d \bar{\lambda}\left(\bar{\lambda}-\eta_{s}\right)\left(\nabla^{2} \psi-\hat{\mathbf{n}} \cdot \nabla(\hat{\mathbf{n}} \cdot \nabla \psi)\right),
\end{aligned}
$$

where $\mathbf{n}=-\hat{\mathbf{e}}_{r}$ refers to the spatial direction of light propagating towards the observer, $\eta_{s}$ is the conformal time at emission, and $\Delta \eta \equiv \eta_{o}-\eta_{s}$. The integrands of the above integrals are taken to depend on $\lambda(\bar{\lambda})$ as well as on $\mathbf{x}=\mathbf{x}_{o}-\hat{\mathbf{n}}\left(\eta_{o}-\lambda\right)$, i.e. they are evaluated on the past light cone. The subscript "o" refers to the observer today.

In Eq. (2) $\eta_{s}$ refers to the background conformal time corresponding to the emission of light from the source. However, in the presence of perturbations a fixed value of $\eta$ does not correspond to a spatially homogeneous value of $z$. Furthermore, it is $z$, not $\eta$, which is measurable and by considering any quantity as a function of redshift in effect one slices the universe in slices of constant $z$, not $\eta$. From Eqs. (56) and (57) of [25] we find for the luminosity distance as a function of redshift the expression:

$$
\frac{d_{L}(z, \hat{\mathbf{n}})}{1+z}-\Delta \eta=\frac{d_{L}\left(\eta_{s}, \hat{\mathbf{n}}\right)}{1+z}-\Delta \eta+\left(\Delta \eta+\frac{1}{\mathcal{H}_{s}}\right)\left[\psi_{s}-\psi_{o}+\left(\mathbf{v}_{o}-\mathbf{v}_{s}\right) \cdot \hat{\mathbf{n}}+2 \int_{\eta_{s}}^{\eta_{o}} d \lambda \hat{\mathbf{n}} \cdot \nabla \psi\right]
$$


from which we obtain

$$
\begin{aligned}
\frac{d_{L}(z, \hat{\mathbf{n}})}{1+z}-\Delta \eta= & \left(2 \Delta \eta+\frac{1}{\mathcal{H}_{s}}\right)\left(\mathbf{v}_{o} \cdot \hat{\mathbf{n}}-\psi_{o}\right)-\left(3 \Delta \eta+\frac{1}{\mathcal{H}_{s}}\right) \mathbf{v}_{s} \cdot \hat{\mathbf{n}}+\left(\Delta \eta+\frac{1}{\mathcal{H}_{s}}\right) \psi_{s} \\
& +2 \int_{\eta_{s}}^{\eta_{o}} d \lambda \psi+2\left(2 \Delta \eta+\frac{1}{\mathcal{H}_{s}}\right) \int_{\eta_{s}}^{\eta_{o}} d \lambda \hat{\mathbf{n}} \cdot \nabla \psi \\
& +2 \int_{\eta_{s}}^{\eta_{o}} d \lambda \int_{\eta_{s}}^{\lambda} d \bar{\lambda} \hat{\mathbf{n}} \cdot \nabla \psi-\int_{\eta_{s}}^{\eta_{o}} d \lambda \int_{\eta_{s}}^{\lambda} d \bar{\lambda}\left(\bar{\lambda}-\eta_{s}\right)\left(\nabla^{2} \psi-\hat{\mathbf{n}} \cdot \nabla(\hat{\mathbf{n}} \cdot \nabla \psi)\right)
\end{aligned}
$$

Eq. (44) can be used to determine the fluctuations of the luminosity distance induced by a Gaussian perturbation [25], such as the one commonly assumed to arise from inflation and describing structure formation in the concordance cosmology. However, we will turn the reasoning the other way round and ask: Assuming the lhs of Eq. (4) is a measured function from SNIa observations and that the underlying model for the background universe is flat and matter dominated with $\Omega_{M}=1$ and $\Lambda=0$, can we determine the perturbation $\psi$ (or $\mathbf{v}$ ) needed to give the observed luminosity distance? In other words, can a local gravitational perturbation fool us into thinking that light has propagated in a universe with the estimated value for the cosmological constant? We will answer this question by assuming that the perturbation $\psi$ exhibits spherical symmetry for simplicity. As discussed in the Introduction, we are interested in the case where the background can still be described by the FRW metric. Furthermore, assuming linear theory, one can relate the velocity field $\mathbf{v}$ with respect to the Einstein-de Sitter background and the Newtonian potential by

$$
\mathbf{v}=-\frac{2}{3 \mathcal{H}} \nabla \psi
$$

Our goal then is to determine $\mathbf{v}$ and $\psi$, given the lhs of (4) from observations (The $d_{L}-z$ relation corresponding to an $\Omega_{\Lambda} \simeq 0.7, \Omega_{M} \simeq 0.3$ universe).

It is convenient to derive a second order equation for the radial velocity $v_{r}$, by acting with two derivatives along the past light cone $\frac{d}{d \eta} \equiv \partial_{\eta}+\hat{\mathbf{n}} \cdot \nabla$, assuming that it is the dominant component. Indeed, if the radial component is to be able to account for the observed $d_{L}-z$ relation, it will have to be be much larger than the other velocity components related to the standard small Gaussian density fluctuations. Given $v_{r}$ the potential can be determined via Eq. (5).

Let us now proceed in the manner just described. Taking two derivatives of (4) we obtain

$$
\frac{d^{2}}{d \eta_{s}^{2}}\left[\frac{d_{L}}{1+z}-\Delta \eta\right]=\left(3 \Delta \eta+\frac{1}{\mathcal{H}_{s}}\right) \frac{d^{2}}{d \eta_{s}^{2}} v_{s r}-\frac{3 \mathcal{H}_{s}}{2}\left(7 \Delta \eta+\frac{13}{3 \mathcal{H}_{s}}\right) \frac{d}{d \eta_{s}} v_{s r}+\frac{15 \mathcal{H}_{s}^{2}}{4}\left(\frac{7}{5} \Delta \eta+\frac{3}{\mathcal{H}_{s}}\right) v_{s r}
$$

where $v_{s r} \equiv-\mathbf{v}_{s} \cdot \hat{\mathbf{n}}$. To derive this result we have used the following: For a matter dominated universe $\frac{d}{d \eta} \frac{1}{\mathcal{H}}=\frac{1}{2}$ and $\partial_{\eta} \psi=0$. The latter relation allows us to write $d \psi / d \eta=\hat{\mathbf{n}} \cdot \nabla \psi=-\frac{3 \mathcal{H}}{2} \hat{\mathbf{n}} \cdot \mathbf{v}$. To eliminate the $\nabla^{2} \psi$ term we assumed that $v_{r}$ is dominant, i.e. that the main component of the peculiar velocity is radial. Thus

$$
\nabla \cdot \mathbf{v}=\left(\frac{2}{r}+\hat{\mathbf{e}}_{r} \cdot \nabla\right) v_{r}=\left(\frac{2}{r}+\frac{\partial}{\partial \eta}-\frac{d}{d \eta}\right) v_{r}
$$

Furthermore,

$$
\frac{3 \mathcal{H}}{2} v_{r}=-\frac{\partial}{\partial r} \psi
$$

Since $\partial_{\eta} \psi=0$, we have that

$$
0=\partial_{\eta}\left(\frac{3 \mathcal{H}}{2} v_{r}\right)
$$

from which we get $(r=\Delta \eta)$

$$
-\Delta \eta \nabla^{2} \psi_{s}=\frac{3 \mathcal{H}^{2}}{2}\left(\frac{\Delta \eta}{2}+\frac{2}{\mathcal{H}}\right) v_{s r}-\frac{3 \mathcal{H} \Delta \eta}{2} \frac{d}{d \eta_{s}} v_{s r} .
$$


We can now express Eq. (6) in terms of the redshift z using

$$
\mathcal{H}=H_{0} \sqrt{1+z}, \quad \text { and } \quad d \eta=-\frac{d z}{H_{0}(1+z)^{3 / 2}} \Rightarrow \Delta \eta=\frac{2}{H_{0}}\left(1-\frac{1}{\sqrt{1+z}}\right) .
$$

We obtain

$$
\left(6-\frac{5}{\sqrt{1+z}}\right)(1+z)^{3} \frac{d^{2} v_{s r}}{d z^{2}}+\frac{3}{2}\left(20-\frac{44}{3 \sqrt{1+z}}\right)(1+z)^{2} \frac{d v_{s r}}{d z}+\frac{3}{4}\left(14+\frac{1}{\sqrt{1+z}}\right)(1+z) v_{s r}=\frac{1}{H_{0}} \frac{d^{2}}{d \eta_{s}^{2}}\left[\frac{d_{L}}{1+z}\right]
$$

This is our main result and can be seen as the equation which defines the perturbation in the (radial) peculiar velocity given an observed $d_{L}-z$ relation, acting as a source on the rhs. Given appropriate boundary conditions, its solution determines the velocity needed to give rise to the observed $d_{L}-z$ curve. We stress again that the rhs is zero for a matter dominated FRW universe and hence any deviation from zero is attributed to a perturbation. Thus, the physically relevant boundary conditions for our case are $v_{r}(z=0)=\frac{d}{d z} v_{r}(z=0)=0$. Allowing for curvature in the background model would bring complications to the perturbation theory and thus would require a separate analysis, so for simplicity and also motivated by the observations we restrict ourselves to the flat model.

Using Eq. (11) we can calculate the rhs of Eq. (12) to find

$$
\frac{1}{H_{0}} \frac{d^{2}}{d \eta_{s}^{2}}\left[\frac{d_{L}}{1+z}\right]=H_{0}\left[(1+z)^{2} \frac{d^{2}}{d z^{2}} d_{L}-\frac{1}{2}(1+z) \frac{d}{d z} d_{L}+\frac{1}{2} d_{L}\right]
$$

Assuming that the observed $d_{L}$ is described by the corresponding theoretical relation for a $\lambda$ CDM universe

$$
d_{L}=\frac{1+z}{H_{0}^{\lambda \mathrm{CDM}}} \int_{1}^{1+z} \frac{d x}{\sqrt{\alpha x^{3}+\beta}},
$$

where $\alpha \simeq 0.3, \beta \simeq 0.7$ and $H_{0}^{\lambda \mathrm{CDM}}=70 \mathrm{~km} / \mathrm{s} / \mathrm{Mpc}$, we have

$$
\frac{1}{H_{0}} \frac{d^{2}}{d \eta_{s}^{2}}\left[\frac{d_{L}}{1+z}\right]=\frac{3}{2} \frac{H_{0}}{H_{0}^{\lambda \mathrm{CDM}}} \frac{(1+z)^{2} \beta}{\left(\alpha(1+z)^{3}+\beta\right)^{3 / 2}} .
$$

In Fig. 1 we plot the solution to the equation of motion for the velocity field, given by Eq. (12), that gives rise to the luminosity distance given in Eq. (15). We also construct the gravitational potential, assuming that $\psi$ is zero at large redshifts. We see that a spherically symmetric linear perturbation can indeed explain the observed acceleration with small velocities and small gravitational potentials. Note that the value $H_{0}$ in the hypothetical $\Omega=1$ dust universe is not really an observable since the model is unlikely to fit all cosmological data. For the purposes of the present approach, it is an adjustable parameter. Here we have chosen $H_{0}=50 \mathrm{~km} / \mathrm{s} / \mathrm{Mpc}$ for illustrative purposes only. As is obvious from the gravitational potential of Fig. 1, physically the perturbation corresponds to a spherical underdensity around us.

The density profile on the light cone can be immediately obtained via the poisson equation

$$
4 \pi a^{2} G \delta \rho=\nabla^{2} \psi-3 \mathcal{H}^{2} \psi .
$$

Using Eqs. (10) and (11) we thus have for the total matter density $\rho=\bar{\rho}+\delta \rho$, where $\bar{\rho}=3 H_{0}^{2} /(8 \pi G)(1+z)^{3}$, along the light cone:

$$
8 \pi G \rho(z)=3 H_{0}^{2}(1+z)^{3}\left[1-\frac{1}{2} \frac{(1+1 / \sqrt{1+z})}{(1-1 / \sqrt{1+z})} v_{s r}(z)-(1+z) \frac{d v_{s r}(z)}{d z}-2 \psi(z)\right] .
$$

In Fig. 2 we plot $\rho(z) / \bar{\rho}_{\text {crit }}(z)$, where $\bar{\rho}_{\text {crit }}(z)=\bar{\rho}(z)=3 H_{0}^{2} /(8 \pi G)(1+z)^{3}$.

\section{TRANSFORMATION TO LTB COORDINATES}

Let us now turn to LTB models. The LTB line-element with the spatial origin at the symmetry center reads as

$$
d s^{2}=-d \tilde{t}^{2}+\frac{\left(A^{\prime}(\tilde{t}, \tilde{r})\right)^{2}}{1-k(\tilde{r})} d \tilde{r}^{2}+A^{2}(\tilde{t}, \tilde{r})\left(d \theta^{2}+\sin ^{2} \theta d \varphi^{2}\right)
$$



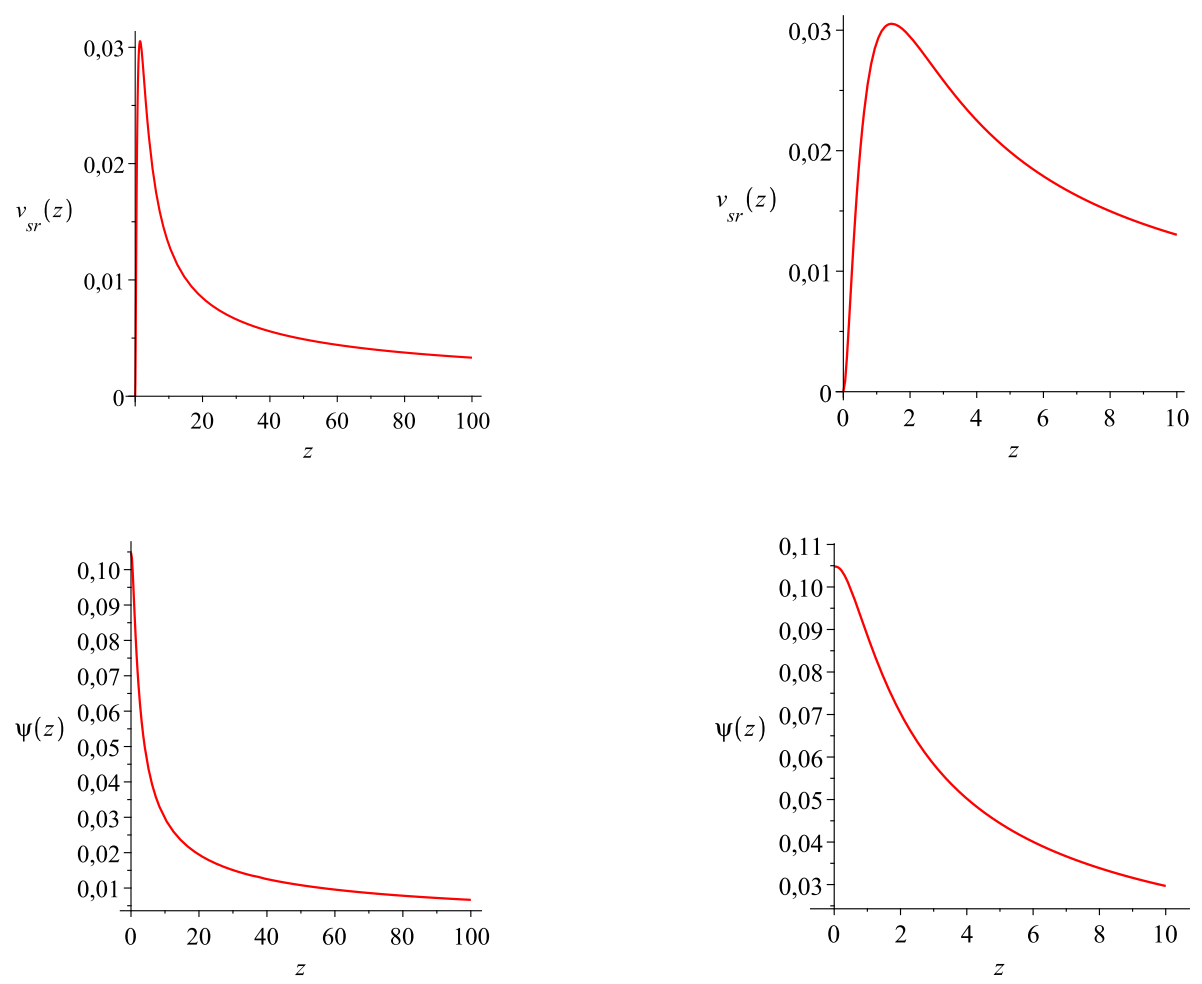

FIG. 1: The velocity field $v_{s r}(z)$ and the gravitional potential $\psi(z)$ of the perturbed FRW universe. The plots on the right are close-ups of those on the left for redshifts $z<10$.
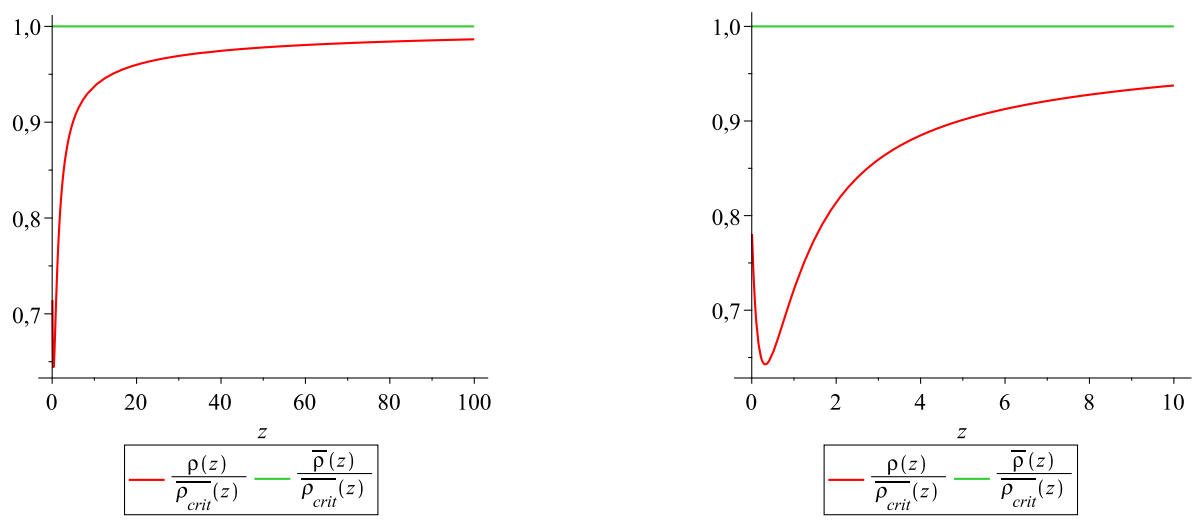

FIG. 2: The density profile $\rho(z) / \bar{\rho}_{\text {crit }}(z)$ (the red line). The green line corresponds to the background Einstein-de Sitter $\bar{\rho}(z) / \bar{\rho}_{\text {crit }}(z)=1$. The plot on the right is a close-up of that on the left for redshifts $z<10$.

where $A(\tilde{t}, \tilde{r})$ is the scale function having both temporal and spatial dependence and $k(\tilde{r})$ is a function associated with the curvature of $\tilde{t}=$ const. hypersurfaces. Both $\tilde{t}$ and $A$ have a direct physical meaning, the first one being the proper time of observers comoving with the dust and the second the physical radius of a sphere with area $4 \pi A^{2}$. The coordinate $\tilde{r}$ is an arbitrary label, devoid of physical meaning. We use the following shorthand notations for the partial derivatives: ${ }^{\prime} \equiv \frac{\partial}{\partial \tilde{r}}$ and ${ }^{\circ} \equiv \frac{\partial}{\partial \tilde{t}}$. The LTB metric (18) is an exact solution of the Einstein equations and the perfectly homogeneous FRW model is a special case, obtained in the limit: $A(\tilde{t}, \tilde{r}) \rightarrow a(\tilde{t}) \tilde{r}$ and $k(\tilde{r}) \rightarrow k \tilde{r}^{2}$, where $a(\tilde{t})$ is the FRW scale factor and $k$ is the curvature constant.

It has been shown that under certain conditions, the LTB metric (18) can be brought to the perturbed FRW form 
(11) via the following nonlinear gauge transformation [10], [22]:

$$
\begin{aligned}
r & =\frac{A(\tilde{t}, \tilde{r})}{a(\tilde{t})}(1+\xi(\tilde{t}, \tilde{r})) \\
t & =\tilde{t}+\xi^{0}(\tilde{t}, \tilde{r})
\end{aligned}
$$

where $a(\tilde{t}) \equiv\left(\tilde{t} / \tilde{t}_{0}\right)^{2 / 3}$ is a fictitious FRW scale factor corresponding to an $\Omega=1$ matter dominated universe. If the LTB model is to be close the the FRW, the functions $\xi(\tilde{t}, \tilde{r})$ and $\xi^{0}(\tilde{t}, \tilde{r})$ must satisfy $|\xi|,\left|\xi^{0} H\right| \ll 1$. We will demonstrate that these conditions are indeed satisfied for our case on our past light cone.

Before proceeding we should note that Eq. (20) provides a relation between the clock times of the LTB and (fictitious) FRW observers. Their difference can be intuitively understood in two ways: In a local description, the LTB coordinates are synchronous, meaning that they are defined by the world-lines of the dust particles which, since pressure is absent, are geodesics. Therefore, their clocks will tick faster than those of any other non-geodesic coordinate system such as the FRW coordinates. The same conclusion is reached in a global description where the LTB spacetime describes a physical underdensity around our position, meaning that the LTB clocks will tick faster with respect to the FRW coordinates in this case. This effect will be more pronounced as we move to the centre and thus $H \xi^{0}$ is expected to decrease with decreasing redshift. Eq. (19) defines the FRW distance $r$ in terms of the physical distance $A$, a prescribed FRW scale factor $a(\tilde{t}) \equiv\left(\tilde{t} / \tilde{t}_{0}\right)^{2 / 3}$ and a function $\xi$ which is chosen to ensure that on the light cone $r$ takes the standard FRW form in terms of redshift:

$$
\frac{A(\tilde{t}(z), \tilde{r}(z))}{a(z)}(1+\xi(\tilde{t}(z), \tilde{r}(z)))=2 H_{0}^{-1}(1-1 / \sqrt{1+z}) .
$$

Furthermore, we choose the arbitrary $\tilde{r}$ such that, on the light cone:

$$
\partial_{\tilde{r}} A(z)=a(z) .
$$

Let us now determine $H \xi^{0}$ and $\xi$. In the LTB model, the angular diameter distance is related to the metric simply by [16]

$$
d_{A}(z)=A(\tilde{r}(z), \tilde{t}(z))=\frac{d_{L}(z)}{(1+z)^{2}}
$$

where the last equality is due to Etherington's theorem [26, 27] stating that for geodesic light in any spacetime the luminosity and angular diameter distances are related through $d_{L}=(1+z)^{2} d_{A}$. If the perturbed FRW and LTB metrics are to describe the same spacetime, just written in different coordinates, the observable distance-redshift relations should be the same: $d_{A}^{\mathrm{LTB}}=d_{A}^{\lambda \mathrm{CDM}}$. So, by having the observable distance-redshift relations we can readily read off $A(\tilde{t}, \tilde{r})$ along the past light cone of the LTB model, that is $A(z)$. $\xi$ can then be determined directly from (21).

Comparing the angular parts of metrics (11) and (18) we see that, to leading order:

$$
A(\tilde{t}, \tilde{r}) \simeq a(t) r[1-\psi(t, r)] \simeq a(\tilde{t}) r\left[1-\psi(\tilde{t}, \tilde{r})+H(\tilde{t}) \xi^{0}(\tilde{t}, \tilde{r})\right],
$$

where we have used (20). In the previous Section we determined the $v(z)$ (or $\psi(z)$ ) profile giving the $\Lambda$ CDM luminosity distance in the perturbed FRW dust universe. Using Eqs. (19), (21) and (23), we can thus obtain the small quantity $H \xi^{0}$ from Eq. (24) along our line of sight:

$$
\begin{gathered}
d_{A}(z)=\frac{2}{H_{0}(1+z)}\left(1-\frac{1}{\sqrt{1+z}}\right)\left(1-\psi(z)+H(z) \xi^{0}(z)\right) \\
\Longrightarrow \quad H(z) \xi^{0}(z)=\frac{d_{A}(z)(1+z) H_{0}}{2\left(1-\frac{1}{\sqrt{1+z}}\right)}+\psi(z)-1,
\end{gathered}
$$

where $d_{A}$ is obtained from Eq. (14) $\left(d_{A}=(1+z)^{-2} d_{L}\right)$.

The outcome is shown in Fig. 33, where we display the shift between $t$ and $\tilde{t}$ in units of the inverse Hubble rate as well as $\xi$. As can be seen both $H \xi^{0}$ and $\xi$ are less than one. In principle, we could have kept higher orders in our expansions, but other than changing the numerical values of $\xi^{0}(z)$ by $\mathrm{O}(30) \%$, we do not expect any qualitative change in this picture. Thus, the conclusion is that an LTB model fitting the SNIa data can be mapped into a perturbed FRW model by a nonlinear coordinate transformation of the form (19), (20), at least on the light cone. 

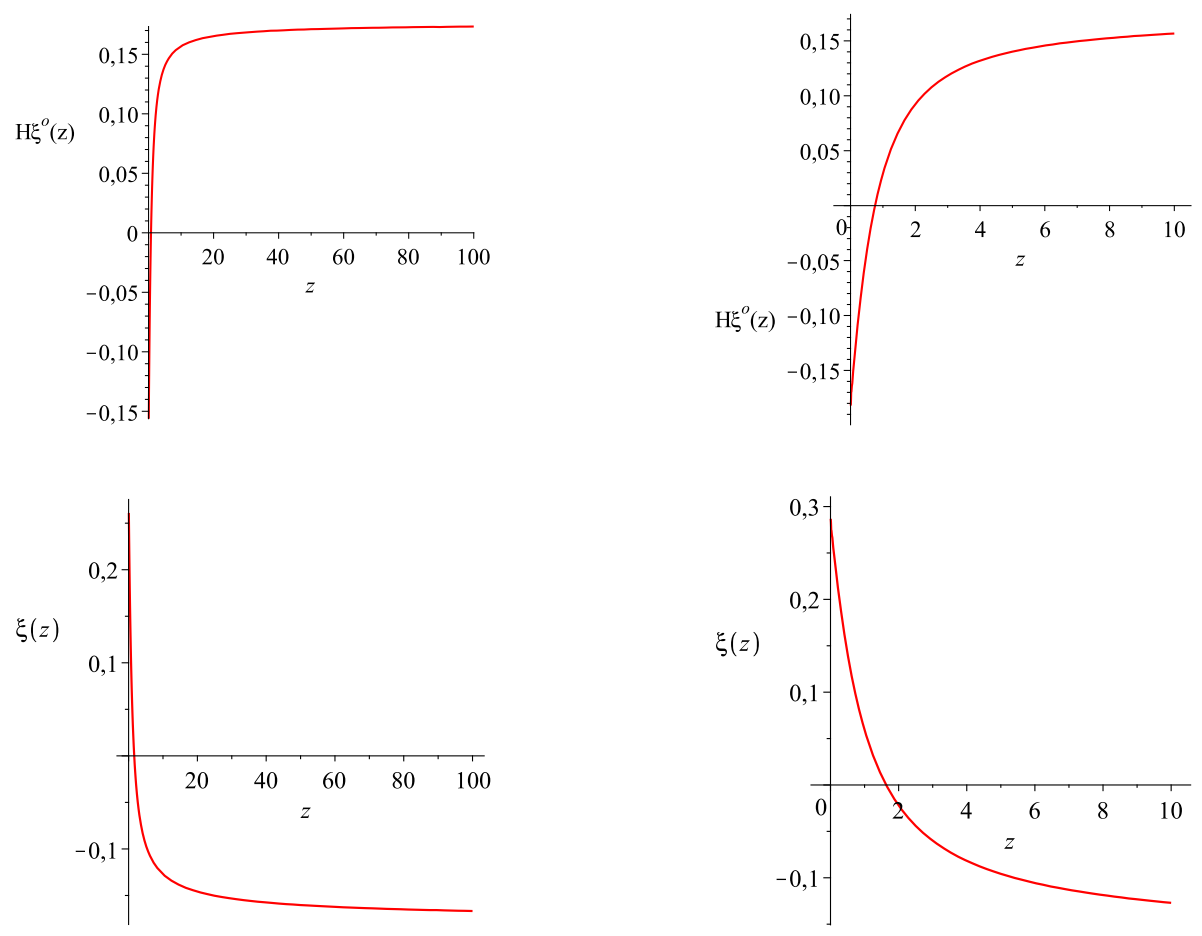

FIG. 3: The functions $H \xi^{0}(z)$ and $\xi(z)$ relating the perturbed FRW and LTB clock times. The plots on the right are close-ups of those on the left for redshifts $z<10$.

In the literature, there has been discussion about the so called "inverse-problem", that is, finding a map from a given luminosity distance to the corresponding LTB model [28, 29, 30]. In fact, in [30] an LTB model, whose $d_{L}-z$ relation is equivalent to that of the $\Lambda \mathrm{CDM}$ model was constructed. Not so surprisingly, the resulting LTB model was found to be such that there is a void whose symmetry center is at the observer's position - assuming the early universe was homogeneous. As expected, our results are consistent with this: In the previous Section we found that the perturbed FRW fitting the $\Lambda$ CDM $d_{L}-z$ curve contains a void around our location, given the universe is asymptotically Einstein-de Sitter. Since the LTB and perturbed FRW are the same spacetime, usually just written in different coordinates, the LTB model must also represent this void. So although we have not explicitly calculated the quantitative properties of the resulting LTB model, the physical content is known from a qualitative point of view and consistent with [30], as it should.

Before summarizing our results, a few comments on the paper [11] are in place, as their results seem to contradict ours. As a toy model, they consider an LTB model consistent with the SNIa data with a large (1 Gpc) void in a universe approaching spatially to Einstein-de Sitter. They find that such models cannot be described in terms of a conformal Newtonian metric perturbed about an FRW background during the entire evolution without large peculiar velocities. However, our result is that the SNIa data can indeed be explained by the perturbed FRW model as well as by an LTB model, with these two descriptions being equivalent and with the peculiar velocity remaining small. Correspondingly, we find that the shifts between FRW and LTB coordinates are also small, in agreement with the analysis of 22] (see also 9]). In their analysis these authors include nonlinear but leading-order terms in the velocities which are absent from [11]. Indeed, since the solution is in the nonlinear regime, one would have to employ the full nonlinear transformation. However, more important is the fact that the peculiar velocities of the model considered in [11] become large at redshifts $z \gtrsim 500$, when the void represents a nonlinear superhorizon density fluctuation, so the model is not even in principle describable within the weak field description of Newtonian gauge. This means that there actually is no discrepancy between their consideration and ours: one can achieve large deviations from the perturbed FRW picture, but only by choosing initial conditions which seem observationally unrealistic. Indeed, when confronted with the CMB observations, a universe dominated by a Gpc sized void seems unlikely. 


\section{DISCUSSION}

We have established that the FRW $\Omega=1$ dust universe with spherically symmetric perturbations in the gravitational potential $\psi(z)$ can fit the SNIa data. We found that physically the perturbation corresponds to a void around us. We do not pretend to know what could be the origin of the perturbation or the associated velocity field, and in fact it is rather obvious that the model as such is likely to be way too simplistic to explain all the relevant cosmological data. However, the interesting point here is that the SNIa data can be explained by small deviations from FRW; both $\psi(z)$ and the velocity field $\mathbf{v}(z)$ remain perturbative at all distance scales. Moreover, such a perturbative approach seems to capture all the essential features of the data. As we demonstrated, if the non-perturbative, spherically symmetric LTB model fits the SNIa data, it can be mapped into the perturbed FRW model by a nonlinear coordinate transformation, at least on the light cone.

Our result seem to contradict [11], where the claim is that the LTB metric cannot be expressed as a perturbed conformal Newtonian metric during the entire evolution of the universe without the existence of a peculiar velocity larger than the speed of light. However, they use a 1 Gpc LTB void model as a tool to demonstrate this and indeed, such case indicates the breakdown of the perturbative description: their model approaches Einstein-de Sitter only spatially and there were strong density contrasts in the early universe. The question is then whether such large nonlinear inhomogeneities are realistic. Observations seem to suggest that the inhomogeneities at the last scattering surface do not lead to such large underdense regions. Our model approaches asymptotically to Einstein-de Sitter along the past light cone, which implies that the early universe was very homogeneous and as our calculations explicitly demonstrate, the explanation of the supernovae data does not require a breakdown of the weak field description. As shown in Fig. 1, the peculiar velocity remains small during the entire evolution along the past light cone and the mapping from LTB to perturbed FRW is possible. This is consistent with the results of [9, 22].

We should like to stress that in fact we have not specified the LTB model quantitatively. That is, we have neither determined the LTB curvature function $k(\tilde{r})$ nor the mass function $F(\tilde{r})$, nor the entire evolution of $A(\tilde{t}, \tilde{r})$ inside our past light cone. However, the underdense bubble around us, represented by the perturbed FRW model, has to be also the physical content of the resulting LTB model from a qualitative point of view since, as we verified, the LTB and perturbed FRW are the same spacetime on our past light cone. In principle, we should have all the information to unambiguously determine also the quantitative properties of that LTB model: Our physical situation is such that the universe approaches asymptotically Einstein-de Sitter along the past light cone, so from this consideration it is obvious that the LTB model contains only growing mode inhomogeneities, so that the early universe was homogeneous and the Big Bang was homogeneous or the age of the universe is independent of the spatial coordinates: $\tilde{t}_{0}(\tilde{r})=$ constant. This requirement sets a constraint between the two boundary condition functions of the LTB model, thus leaving only one free function and a free parameter $\tilde{t}_{0}$, just like in the perturbed FRW case - the potential $\psi(z)$ (or alternatively, the velocity field $\mathbf{v}(z))$ and a free parameter $H_{0}$. However, our interest here was not to concentrate on the exact model building. In fact, the issue of finding an LTB model that fits to a given $d_{L}-z$ curve has been previously addressed by a few authors [28, 29, 30, 31] and indeed, the physical content of the LTB model described here is consistent with the result obtained in [30], where an LTB model was constructed by demanding its luminosity distance to be equivalent with the one in the $\Lambda \mathrm{CDM}$ model - their result is a void, the center of which we occupy. We hope to return to the issue of quantitatively determining the LTB model from luminosity distance data in future work. It will also be interesting to see whether the perturbative nature of the model persists inside the past light cone.

We thus conclude that the SNIa data does not require a deviation from the Copernican Principle that is nonperturbative, at least if we limit ourselves to spherically symmetric perturbations. Indeed, it would be of some interest to generalize the present approach to non-spherical perturbations; whether this can be done in practice remains to be seen.

\section{Acknowledgements}

This work was partly supported by the EU 6th Framework Marie Curie Research and Training network "UniverseNet" (MRTN-CT-2006-035863) and partly by Academy of Finland grant 114419. MM is supported by the Graduate School in Particle and Nuclear Physics. MM wishes to thank Teppo Mattsson for useful discussions. 
[1] R. R. Caldwell and A. Stebbins, Phys. Rev. Lett. 100 (2008) 191302 arXiv:0711.3459 [astro-ph]].

[2] C. Clarkson, B. Bassett and T. C. Lu, Phys. Rev. Lett. 101 (2008) 011301 arXiv:0712.3457 [astro-ph]].

[3] J. P. Uzan, C. Clarkson and G. F. R. Ellis, Phys. Rev. Lett. 100 (2008) 191303 [arXiv:0801.0068 [astro-ph]].

[4] S. Perlmutter et al. [Supernova Cosmology Project Collaboration], Astrophys. J. 517 (1999) 565 arXiv:astro-ph/9812133. A. G. Riess et al. [Supernova Search Team Collaboration], Astron. J. 116 (1998) 1009 arXiv:astro-ph/9805201.

P. Astier et al. [The SNLS Collaboration], Astron. Astrophys. 447 (2006) 31 arXiv:astro-ph/0510447].

A. G. Riess et al., Astrophys. J. 659 (2007) 98 arXiv:astro-ph/0611572.

[5] T. Buchert, Gen. Rel. Grav. 32 (2000) 105 arXiv:gr-qc/9906015.

[6] T. Buchert, Gen. Rel. Grav. 40 (2008) 467 arXiv:0707.2153 [gr-qc]].

[7] S. Rasanen, JCAP 0611 (2006) 003 arXiv:astro-ph/0607626.

[8] S. Rasanen, JCAP 0804 (2008) 026 arXiv:0801.2692 [astro-ph]].

[9] A. Paranjape and T. P. Singh, Phys. Rev. Lett. 101 (2008) 181101 arXiv:0806.3497 [astro-ph]].

[10] A. Paranjape and T. P. Singh, JCAP 0803 (2008) 023 arXiv:0801.1546 [astro-ph]].

[11] E. W. Kolb, V. Marra and S. Matarrese, Phys. Rev. D 78 (2008) 103002 arXiv:0807.0401 [astro-ph]].

[12] T. Mattsson and M. Mattsson, JCAP 0802 (2008) 004 arXiv:0708.3673 [astro-ph]].

[13] T. Mattsson, arXiv:0711.4264 [astro-ph].

[14] G. Lemaitre, Annales Soc. Sci. Brux. Ser. I Sci. Math. Astron. Phys. A 53 (1933) 51.

R. C. Tolman, Proc. Nat. Acad. Sci. 20 (1934) 169.

H. Bondi, Mon. Not. Roy. Astron. Soc. 107 (1947) 410.

[15] A. Krasinski, "Inhomogeneous Cosmological Models", Cambridge University Press (1997).

[16] J. Plebanski, A. Krasinski, "An Introduction to General Relativity and Cosmology", Cambridge University Press (2006).

[17] For a recent review of LTB models, see K. Enqvist, Gen. Rel. Grav. 40, 451 (2008) arXiv:0709.2044 [astro-ph]].

[18] M. N. Celerier, Astron. Astrophys. 353 (2000) 63 arXiv:astro-ph/9907206].

H. Iguchi, T. Nakamura and K. i. Nakao, Prog. Theor. Phys. 108 (2002) 809 arXiv:astro-ph/0112419.

D. Garfinkle, Class. Quant. Grav. 23 (2006) 4811 arXiv:gr-qc/0605088.

D. J. H. Chung and A. E. Romano, Phys. Rev. D 74 (2006) 103507 arXiv:astro-ph/0608403.

K. Enqvist and T. Mattsson, JCAP 0702 (2007) 019 arXiv:astro-ph/0609120.

[19] K. Tomita, Astrophys. J. 529 (2000) 26 arXiv:astro-ph/9905278.

K. Tomita, Prog. Theor. Phys. 106 (2001) 929 arXiv:astro-ph/0104141].

K. Tomita, Mon. Not. Roy. Astron. Soc. 326 (2001) 287 arXiv:astro-ph/0011484].

H. Alnes, M. Amarzguioui and O. Gron, Phys. Rev. D 73 (2006) 083519 arXiv:astro-ph/0512006.

H. Alnes and M. Amarzguioui, Phys. Rev. D 74 (2006) 103520 arXiv:astro-ph/0607334.

[20] A. Ishibashi and R. M. Wald, Class. Quant. Grav. 23 (2006) 235 arXiv:gr-qc/0509108.

[21] A. Paranjape, arXiv:0906.3165 [astro-ph.CO].

[22] K. Van Acoleyen, JCAP 0810, 028 (2008) arXiv:0808.3554 [gr-qc]].

[23] M. Sasaki, Mon. Not. Roy. Astron. Soc. 228 (1987) 653.

[24] E. Barausse, S. Matarrese and A. Riotto, Phys. Rev. D 71 (2005) 063537 arXiv:astro-ph/0501152.

[25] C. Bonvin, R. Durrer and M. A. Gasparini, Phys. Rev. D 73 (2006) 023523 arXiv:astro-ph/0511183.

[26] I. M. H. Etherington, Phil. Mag. ser. 7, 15 (1933) 761.

[27] G. F. R. Ellis, "Relativistic Cosmology", p. 104 in Proc. School Enrico Fermi, "General Relativity and Cosmology", Ed. R. K. Sachs, Academic Press (New York 1971).

[28] H. Iguchi, T. Nakamura and K. i. Nakao, Prog. Theor. Phys. 108 (2002) 809 arXiv:astro-ph/0112419.

[29] D. J. H. Chung and A. E. Romano, Phys. Rev. D 74 (2006) 103507 arXiv:astro-ph/0608403.

[30] C. M. Yoo, T. Kai and K. i. Nakao, Prog. Theor. Phys. 120 (2008) 937 [arXiv:0807.0932 [astro-ph]].

[31] N. Mustapha, C. Hellaby and G. F. R. Ellis, Mon. Not. Roy. Astron. Soc. 292 (1997) 817 arXiv:gr-qc/9808079]. 